\title{
UNDERSTANDING PARAMETRIC RESONANCE EFFECTS IN COMMON MEM ACTUATORS
}

\author{
Kimberly L. Turner and Noel C. MacDonald \\ Department of Mechanical \& Environmental Engineering \\ University of California, 2355 Engineering Building II, Santa Barbara, CA 93106 \\ Phone: (805) 893-5106, Fax: (805) 893-8651, email: turner@engineering.ucsb.edu
}

\begin{abstract}
Parametric resonance has been previously observed in electrostatically actuated torsional microelectromechanical systems (MEMS) [1], but it can occur in other common MEMS devices, such as in-plane resonators which use non-overlapping comb drives or parallel-plate drives. Parametric resonance can occur whenever the force generated by a periodically-driven actuator changes with position, characteristically creating very large amplitude responses at specific frequencies. In this paper, two distinct actuator types are analyzed, and experimental results are shown. Parametric resonance has been verified for a nonoverlapping comb drive actuator, and the fundamental differences between a parametrically excited state and a directly forced state are shown. We present a straightforward way of determining if parametric resonance is present in a MEM system.
\end{abstract}

\section{INTRODUCTION}

Deviations from 'expected' or 'designed' behavior is one mechanism of failure in MEMS devices. Failure can come from material fatigue, defects, or incorrect operation but also from system dynamics which are not accounted for in many simple models. There are certain regimes where the device can strongly deviate from linear approximations often used for design. Parametric resonance is just one such event which can occur, and cause device behavior to strongly deviate from the equations often used in design. Parametric resonance has been theoretically studied [2,3] for many years, and has been observed in the forced motion of a swing, stability of ships, Faraday crispations, along with others. Theory predicts that parametric resonances occur near drive frequencies which are certain fractions of the drive frequency. Thus the drive frequency can be a value far removed from resonance, yet still lead to a large amplitude, 'resonant' type of response from the device. Thus the phenomenon must be clearly understood, so that it can be utilized or avoided as necessary to maintain desired performance.

\section{THEORY}

In the systems discussed here, parametric resonances occur when [2]:

$$
\omega=\omega_{d} / n(\mathrm{n}=1,2,3 \ldots)
$$

When designing actuators to operate at or near resonance, it is important to understand how parametric resonance will affect the device operation, as it can occur at the same drive frequency as a standard resonant response.

\section{PARALLEL PLATE ACTUATOR}

Parametric resonance has been observed and studied in a variety of structures. One such structure is the well-known parallel-plate actuator (see Figure 1).

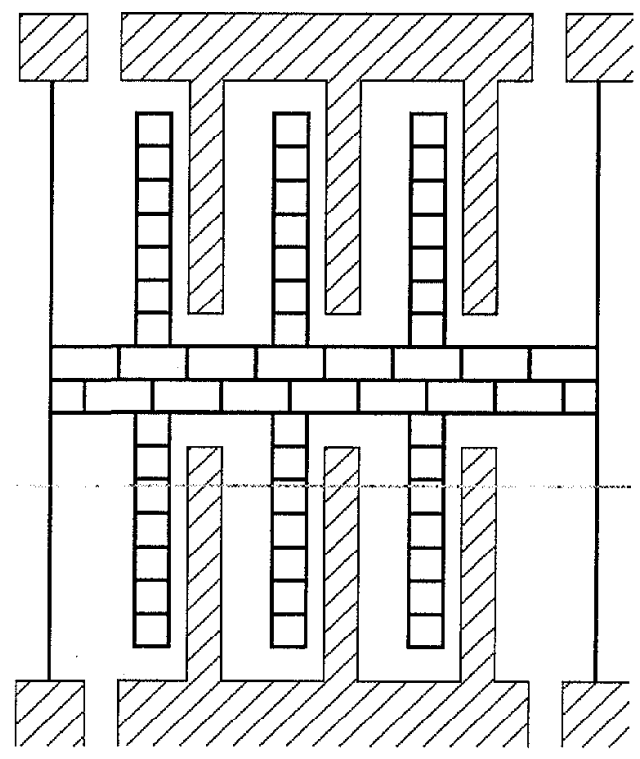

Figure 1. Schematic of a MEMS oscillator with parallel-plate capacitors for actuation. The equation of motion for such an oscillator is given by Equation 1 , where $m$ is the mass, $c$ is a damping coefficient, $k$ is a linear stiffness term, $A$ is the area of overlapping plates, $d_{o}$ is the initial gap between movable and fixed plate, and $\omega$ is the drive frequency.

The equation of motion of a parallel-plate capacitor when sinusoidally driven is (assuming linear springs and linearizing the parallel plate force):

$m \ddot{x}+c \dot{x}+\left(\left(k-\frac{\varepsilon_{o} A V_{a c}^{2}}{2 d_{o}^{3}}\right)+\frac{\varepsilon_{o} A V_{a c}^{2}}{2 d_{o}^{3}} \cos (2 \omega t)\right) x=\frac{\varepsilon_{o} A V_{a c}^{2}}{4 d_{o}^{2}}(1-\cos 2 \omega t)$

(variables defined in Figure 1 , and $\mathrm{V}_{\mathrm{ac}}=\mathrm{AC}$ drive amplitude, $\varepsilon_{0}=$ permittivity constant). Through numerical simulation, it is determined that the parametric forcing term has a large effect on this system. Figure 2 shows the normalized $(\tau=\omega t)$ response of an equation driven at $\omega=0.5 \omega_{o}(n=2$, see Equation 1) with the parametric forcing term set equal to zero. Figure 3 shows the response of the normalized equation with a nonzero parametric forcing term. Note the differences between the two responses. Figure 2 displays a linear growth, which is expected for an externally forced system, whereas Figure 3 displays an exponential 
growth, common to parametric resonance $[2,3]$. This behavior can be easily measured using motion characterization techniques available for MEMS.

Damping and applied signal strength have a significant effect on parametric resonant response, although not through amplitude. Primary $(n=1)$ parametric resonance can only occur if the drive strength term, $\delta$ (in this case, $\delta=\frac{\varepsilon_{o} A V_{a c}^{2}}{4 \omega^{2} d_{o}^{3}}$ ) is greater than $2 \mu$, where $\mu=c / 2 \omega$. For $n=2, \delta^{2}>16 \mu$. Thus, if parametric resonance is not desired, it can be avoided by adjusting the drive strength and/or operating environment according to the above condition.

Parametric resonance has been observed in parallel-plate actuators, novel comb actuators, and torsional resonators. Using precise experimental testing techniques, it is now straightforward to distinguish between parametric resonance and directly forced resonance in MEMS devices. Understanding the dynamics of resonant MEMS leads to more intelligent, robust designs and a greater range of applications which can be developed.

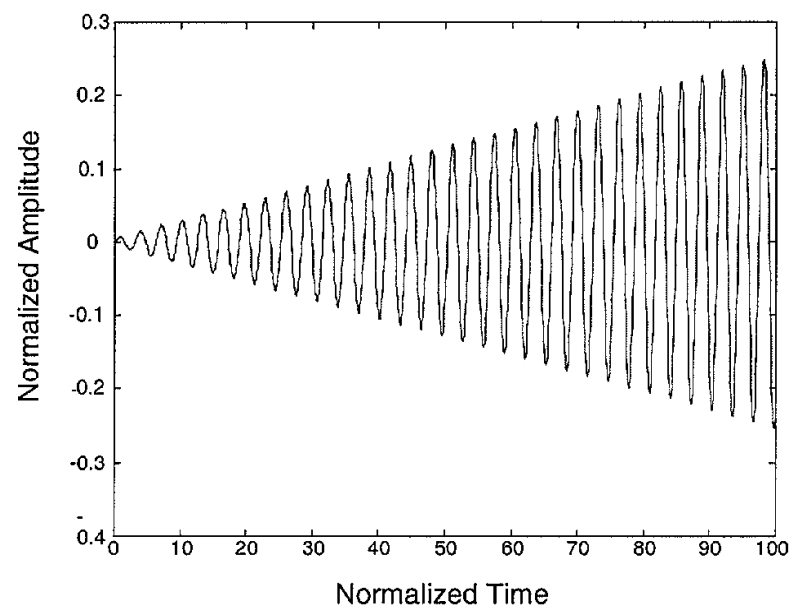

Figure 2. Normalized resonant response of a non-parametric resonator. Note the linear growth in amplitude inherent in undamped second-order $O D E$ 's. The normalized equation solved was: $\ddot{x}+\beta x=e \cos 2 \tau$, where $\beta=4, e=0.01$

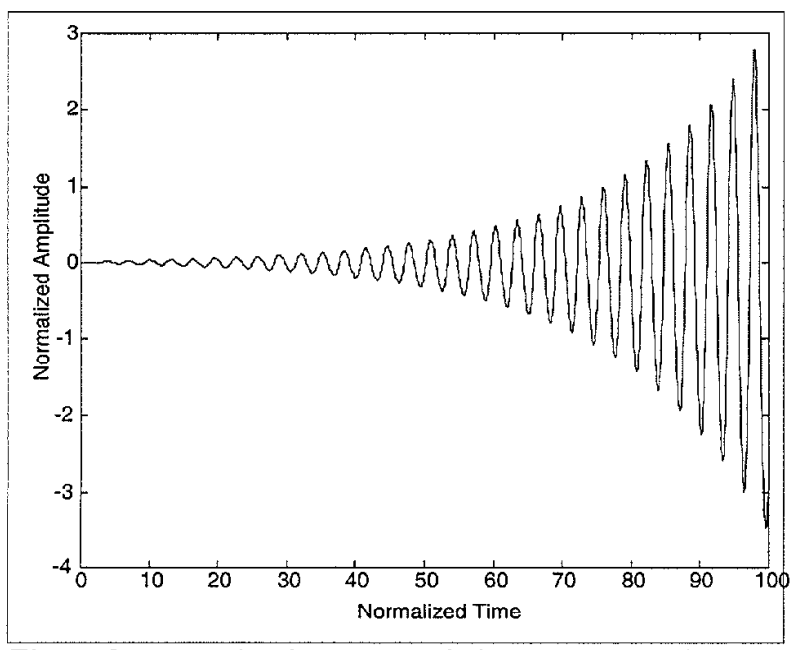

Figure 3. Normalized response of above system, with parametric driving term: $\ddot{x}+(\beta+2 \delta \cos 2 \tau)=e \cos 2 \tau$, where $\beta=4, \delta=0.5$, and $e=0.01$. Note the exponential growth present due to the parametric term. Also note the larger amplitude obtained within the same time.

\section{NON-OVERLAPPING COMB DRIVE ACTUATORS}

Other novel actuator designs have been developed which display parametric resonance behaviors. Some important actuators which display this behavior are the novel fringing-field actuators which were designed by Adams, et. $\mathrm{Al}$ [4] for the independent tuning of linear and cubic stiffness terms [4,5]. The actuators resemble comb drive actuators, except that the fingers are not interdigitated. Figure 5 shows a diagram of the actuator configurations. The top of the figure shows the reduction configuration (if the actuator moves to the left, the net force toward the left is increased), while the bottom describes the augmentation configuration (if the actuator moves to the left, the net electrostatic force is toward the right). These actuators, unlike standard comb-drive actuators, move perpendicular to the direction of the fingers, as shown schematically in the figure. They move due to fringing electric fields between the movable and fixed fingers.

Depending on the original position of the movable fingers with respect to the fixed fingers, the electrostatic spring constant acts negative or positive. A schematic showing the force vs. displacement is shown in Figure 5. This graph shows the general trend of this type of actuator. Specific simulations and experiments of force vs. displacement relations for this type of actuator have been performed by Adams et. Al. [5]. If you examine Figure 5, it is easy to identify two distinct linear regions. In the region surrounding points marked by the open squares, the curve has a negative slope (augmentation), and for the region surrounding points marked by open circles (reduction), the curve has a positive slope. Thus, depending on the initial position of the actuator, the behavior of the device can be quite different.

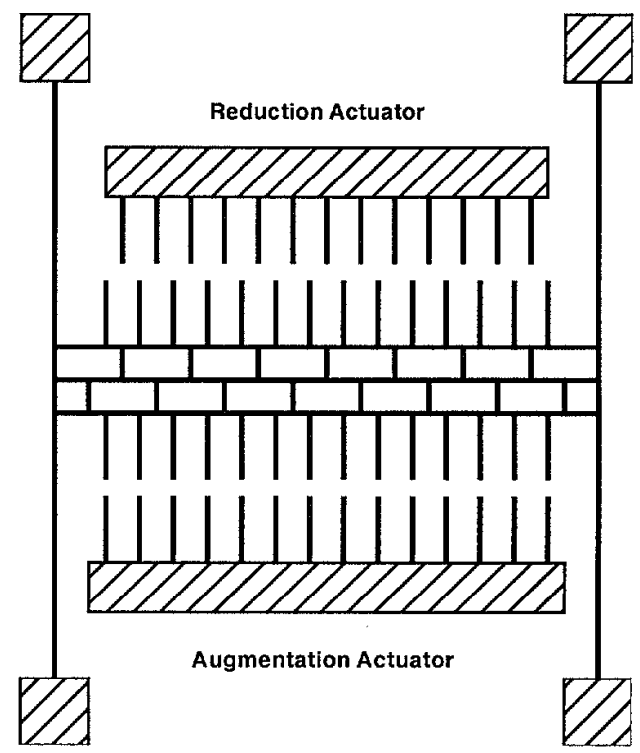

Figure 4. Schematic of a non-overlapping comb drive actuator, suspended from fixed-fixed springs. 


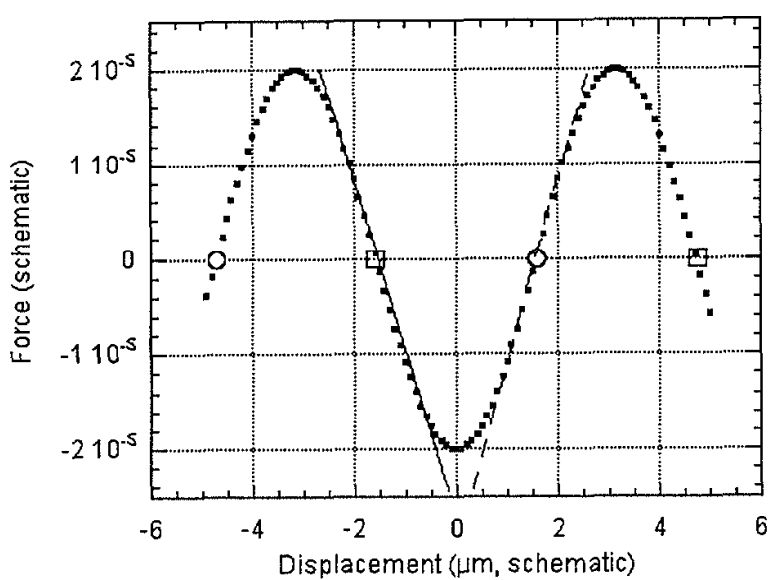

Figure 5. A schematic of the force curve showing different regions of the force relation for reduction and augmentation actuators. The open squares represent centers of the augmentation regions, while the open circles represent centers of the reduction regions.

$\Lambda \mathrm{n}$ approximate equation of motion for each of these regions can be developed. Beginning with the standard equation of motion, and assuming that the devices have a fixed-fixed spring configuration, with mechanical stiffnesses which are linear with a small cubic term, we get:

$$
m \ddot{x}+c \dot{x}+k x+h x^{3}=F(x, t)
$$

where $F$ depends on whether a reduction or augmentation configuration is used. First, consider the augmentation alignment, where the slope of $F$ vs. position is negative. A sinusoidal driving term is applied, although a square-rooted sinusoid as developed by Turner et. Al. [1] can also be applied. For a sinusoidal driving term, the equation of motion becomes:

$$
m \ddot{x}+c \dot{x}+k x+h x^{3}=-\xi x(V \sin \omega t)^{2}
$$

and if $V^{2} \sin ^{2} \omega t$ is represented by trigonometric substitution as $V^{2}(1-$ $\cos 2 \omega t) / 2$, the equation becomes:

$$
\ddot{x}+c / m \dot{x}+1 / m\left[\left(k+\frac{V^{2}}{2} \xi\right)-\frac{V^{2}}{2} \xi \cos 2 \omega t\right] x+\frac{h}{m} x^{3}=0
$$

which is a parametrically forced equation of the Mathieu type. When applying the same theory as presented in Chapter 2 , instabilities are predicted where $\omega=\omega_{0} / n$, where $\left.\omega_{0}{ }^{2}=(1 / m)\left(k+V^{2} \xi / 2\right)\right)$. A small DC offset may be present in the input signal which can lead to a small term on the right hand side of the equation. Thus as in the parallel plate equation, there can be a small direct driving term, which can dominate at times in the response.

The reduction actuator has the same form as Equation 4, except the sign is reversed on the term proceeding $x$ due to electrostatic forcing. Thus, the equation of motion appears as:

$$
\ddot{x}+c / m \dot{x}+1 / m\left[\left(k-\frac{V^{2}}{2} \xi\right)+\frac{V^{2}}{2} \xi \cos 2 \omega t\right] x=0
$$

which is a parametrically forced differential equation of Mathieu type, and respond as previously discussed. This equation also predicts instabilities at frequencies at $\omega=\omega_{\delta} / n$.

\section{EXPERIMENT}

The device shown in Figure 6 is a single-crystal Silicon MEMS actuator fabricated using the SCREAM process. It has comb drives on either end, and along the backbone, it has non- overlapping comb drives, as shown schematically in Figure 2. The non-overlapping comb drives are used for this experiment, thus the equation of motion is similar to Equation 6. As seen in theory, the growth rate and envelope are distinctly different depending on whether parametric resonance is the primary reason for the amplitude growth. Thus by capturing this growth region, it can be determined if parametric resonance is present, and if it has a strong effect on the device behavior.

Using an oscilloscope, the time-series behavior can be captured. The motion is measured using a laser doppler vibrometer coupled through an optical microscope [6]. The device is in a pressure-controlled chamber. Figure 7 shows the input signal which produced the response in Figure 8. This input signal was at $\sim 13 \mathrm{kHz}$. The resonant frequency of the device is at $\sim 26 \mathrm{kHz}$. Figure 8 shows an experimental response typical of nonparametric oscillation. The response has a growth rate typical of an underdamped oscillator. The amplitude is limited in this case by both damping and nonlinearity [7].

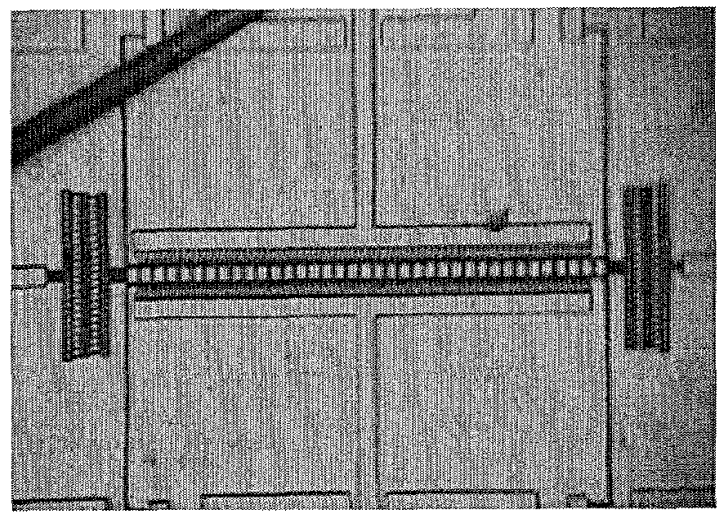

Figure 6. Device with novel comb actuators used for the experiments in this device. This device is being driven by actuators as shown in Figure 2, which are non-overlapping comb drives.

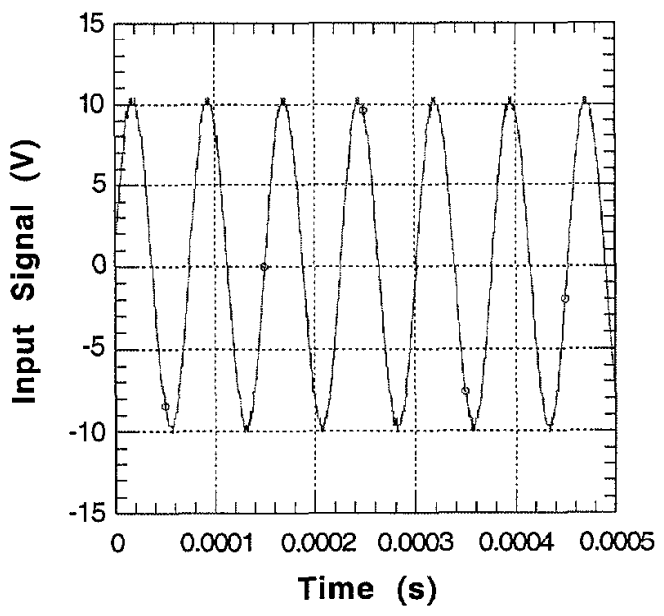

Figure 7. Input signal to the device at $13 \mathrm{kHz}$. This response is dominated by direct forcing, and does not display parametric resonance behavior, as shown below. Parametric effects only occur at higher orders $(n>1)$ in the presence of very low damping. Thus at this frequency, (corresponding to $n=2$ ) the parametric resonance response is not present. 


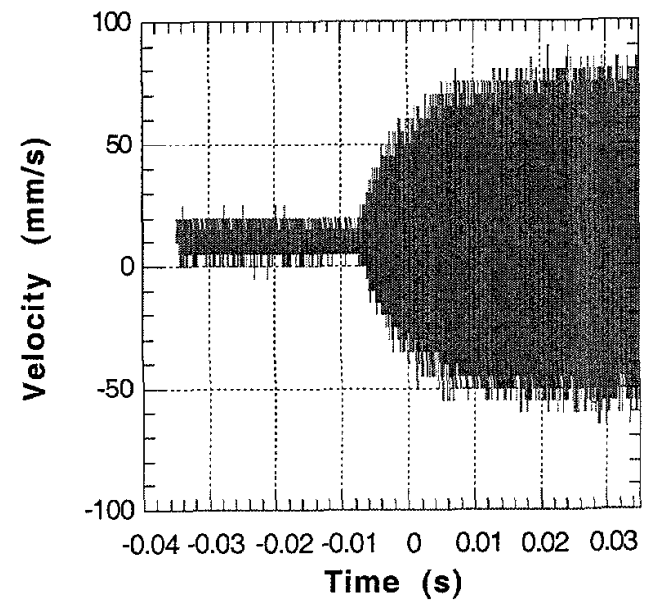

Figure 8. Turn on data when driving at a frequency which does not have a large parametric frequency response. As seen, this device has a response typical of a damped, driven oscillator. The limiting amplitude is primarily due to the damping present. The device was at $200 \mathrm{~m}$ Torr under test.

Directly forced oscillations are primarily limited by damping, which is not the case for parametric resonance. Thus, in some cases the parametric oscillations can lead to larger amplitude motions than direct resonance.

Figure 9 shows the input signal used to obtain the response curve shown in Figure 10. In this case, the device was driven at $\omega=\omega_{0}$, corresponding to the $n=1$ parametric instability region. This is the strongest parametric oscillation, and it is apparent from the exponential growth behavior that the response is largely due to parametric resonance.

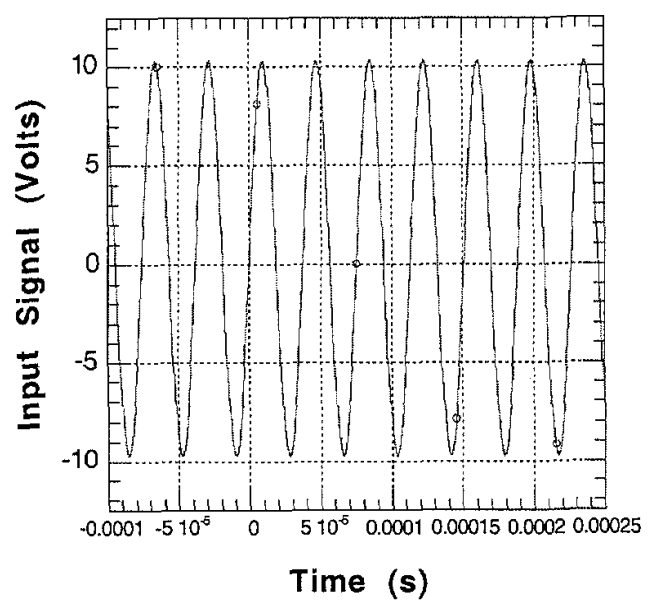

Figure 9. Input signal which produced the parametric resonance curve shown below. As seen in the simulations, the behavior strongly suggests a parametric resonant type of behavior.

The amplitude reaches a maximum which is not predicted by the linear theory. This maximum value is caused by a nonlinearity, not damping as in the previous case [7]. The nonlinearity can come from electrostatics as well as geometric nonlinearity, and is currently being investigated.

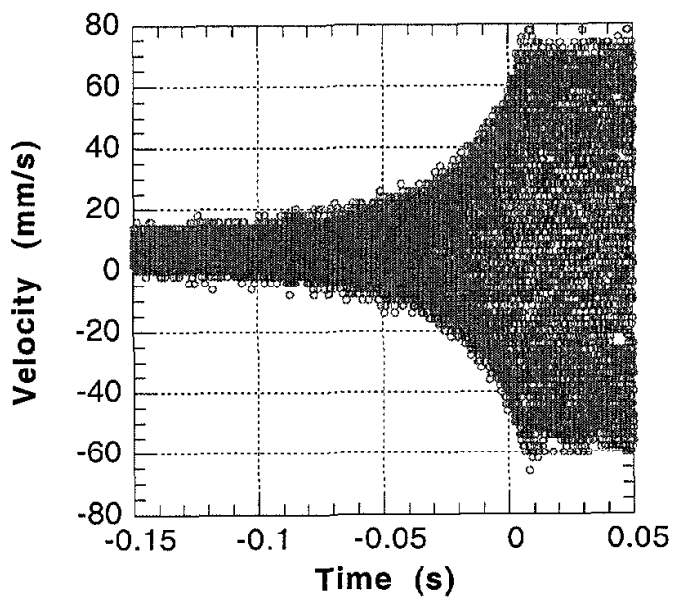

Figure 10. Turn-on data when driving in Parametric resonance $n=1$ instability region. Note the exponential growth behavior as predicted in numerical simulation, and also by perturbation theory. The pressure was $\sim 100$ mTorr for this experiment.

\section{CONCLUSION}

As shown by theory as well as simulated and experimental results above, large amplitude responses can appear at frequencies other than the first natural frequency of common MEM devices. These behaviors are present in many types of MEM actuators, including non-overlapping comb drives, parallel plate actuators, and torsional actuators. Understanding and having simple tests to determine which type of resonance is present is key to understanding and designing robust and predictable MEMS for many applications.

\section{ACKNOWLEGDMENTS}

The authors would like to acknowledge Scott G. Adams and Russ Webb for samples used in this paper. The fabrication of devices for this paper was performed at the Cornell Nanofabrication Facility. This research was sponsored by DARPA contract \# DABT63-95-C-0121 and the University of California, Santa Barbara.

\section{REFERENCES}

[1] Turner, K. L. et. Al., "Five parametric resonances in a microelectromechanical system," Nature, 396, 149-153 (1998).

[2] Cunningham, W. J ., Introduction to Nonlinear Analysis McGraw Hill, Inc., New York, 1958.

[3] Bolotin, V. V., The Dynamic Stability of Elastic Systems. Holden-Day, San Francisco. (1964).

[4] Adams, S. G., et. Al., "Independent Tuning of Linear and Nonlinear Stiffness Coefficients," Journal of Microelectromechanical Systems, 7, 2 (1998).

[5] Adams, S. G., "Capacitance based tunable resonators," Joumal of Micromechanics and Microengineering, 8, 15-23 (1998).

[6] Turner, K. L., P. G. Hartwell, and N. C. Macdonald, "Three dimensional motion characterization using Laser Vibrometry," Transducers '99, Sendai, Japan, 7-10 June 1999, pp. 1144-1147. [7] Nayfeh, A. H. and D. T. Mook, Nonlinear Oscillations, John Wiley and Sons, New York (1979). 